\title{
is Research Suare \\ Genomic Diversity and Spatiotemporal Distributions of Lassa Virus Outbreaks in Nigeria
}

\section{Abdulwahid Abaukaka Yahaya}

Tehran University of Medical Sciences

\section{Yazdan Asgari ( $\nabla$ yasgari@tums.ac.ir)}

Tehran University of Medical Sciences https://orcid.org/0000-0001-6993-6956

\section{Research article}

Keywords: Genomic, Lassa Virus, Phylogeography, Spatiotemporal and Nigeria

Posted Date: October 21st, 2019

DOI: https://doi.org/10.21203/rs.2.16266/v1

License: @ (i) This work is licensed under a Creative Commons Attribution 4.0 International License. Read Full License 


\section{Abstract}

Background Lassa virus (LASV) is a single-negative strand RNA Arenavirus (genus Mammarenavirus ), oriented in both negative and positive senses. Due to the increase in fatality rate of deadly disease LASV caused (Lassa fever), widespread of LASV in Nigeria has been a subject of interest. Following the upsurge of LASV endemicity in 2012, another marked incidence were recorded in Nigeria, 2018, with 394 confirmed cases in 19 states, and estimated $25 \%$ cases led to death. This study aimed at acquiring the genetic variation of LASV ancestral evolution with evolvement of new strains in different lineage and its geographical distributions within specific time of outbreaks through Bayesian inference, using genomic sequence across affected states in Nigeria.

Results From the result, we were able to establish the relationship of Lassa mamarenavirus and other arenaviruses by classifying them into distinct monophyletic groups, i.e. the old world arenaviruses, new world arenaviruses, and Reptarenaviruses. Corresponding promoter sites for genetic expression of the viral genome were analyzed based on Transcription Starting Site (TSS), the S_Segment (MK291249.1) is about 2917-2947 bp and L_Segment (MH157036.1), is about1863-1894 bp long. LASV sequence obtained from different part of Nigeria were genetically related. Benue, Imo, and Bauchi states represent the host etiology of the LASV. Spread across other neighboring states were based on genetic pedigree dated to previous outbreaks as at year 2008 to 2012. Phylogeography of recent transmission from year 2017 to 2019 indicates vectors were fast spreading LASV from Ondo states to Delta, Edo, and Kogi states, while spread across north eastern states suggests a vector origin from Bauchi state.

Conclusions The study evidently outlined the path of transmission based on genetic homology of the sampled LASV sequences in affected geographical locations. we suggests, federal government should initiate a vector surveillance program to curtails further spread of LASV, especially, states bordering with north western states and north central Nigeria.

Keywords Genomic, Lassa Virus, Phylogeography, Spatiotemporal and Nigeria

\section{Background}

Based on clinical investigation, Lassa virus (LASV) was endemic in several countries of West Africa, Nigeria, Sierra Leone, Guinea, and Liberia[1]. Cases of LASV were also reported in Senegal, Gambia, Mali, Burkina Faso, Ivory Coast and Ghana [1-3]. LASV is an Arenavirus with segmented negative-strand RNA, oriented in both negative and positive senses (ambisense gene coding) on the two RNA segments[4]. LASV genome is comprise of two genetic segments, $L(7.3 \mathrm{~kb})$ and $S(3.4 \mathrm{~kb})$, encode four proteins: $Z$ protein, $L$ protein, nucleoprotein, and glycoprotein [5]. The RNA polymerase L protein (200 kDa) and a RING finger Z protein $(11 \mathrm{kDa})$ are coded in $\mathrm{L}$ segment, while the $S$ segment code for nucleoprotein $(64 \mathrm{kDa})$ and two cleaved glycoproteins GPC1 (42 kDa) and GPC2 (38 kDa)[6]. Currently, 35 species including Lassa mammarenavirus are carried by mammalian host and they are categorized into two main groups, Old World and New World viruses[7]. This zoonotic disease which pose a greater threat to public health further classified into virus subgroups based on phylogenetic, serological, and geographical characteristics; the 
Old World viruses, comprises of LASV and Lujo virus (LUJV), while the New World viruses includes Machupo and Chapare virus from Bolivia, Junín virus, Sabiá virus and Guanarito virus found in Argentina, Brazil and Venezuela, respectively[8]. Genomic studies of LASV can enhance the acquisition of data-driven distribution and determinant of the Lassa fever disease by defining genetic variations and viral specific lineage [9].

Out of all LASV proteins that exist in the virions and infected host cells, nucleoprotein has the largest number of polypeptide encapsidated genomic RNA to prevent it from degradation in infected host cell [10]. LASV nucleoprotein is made up a peptide having 569 amino acids residue [11] and consist of separate amine group (N-terminal) and carboxylic group (C-terminal) domains [12]. The $\mathrm{N}$-terminal domain proposed a cap-binding cellular structure through deep binding cavity for the synthesis of viral mRNA[13], while the C-terminal domain revealed a binding site functions as exoribonuclease, leading to suppression of type I IFN production by interfering with IFN regulatory factor 3 (IRF-3) activation $[12,14,15]$. This pathway plays important role in transcription and replication of Lassa virus and immunosuppression of infected host $[11,16]$

LASV glycoprotein (GP) is a trimeric single polypeptide chain, glycosylated cotranslationally, cleaved subunit GP-1 and GP-2 by enzyme peptidase in the endoplasmic reticulum (ER) which play important role in viral-host infection $[17,18]$ Glycoprotein facilitates the entry of the virion into the host cell through receptor binding and fusion to the cell membrane as the only unique protein of the capsid that protect humoral immunity [17]. Prior to the GP-1 attachment to the host cells, there is a low immune response that poses a major problem to the host immune system as the virus spike in response to protective antibodies. This is due to the extensive protection by N-linked glycan, a phenomena which is similar to other infectious diseases caused by viruses such as human immunodeficiency virus and hepatitis C virus [19]. Unlike GP1, the fusion of GP-2 facilitates by internal fusion loop (I-FP) that trigger the transmembrane fusion as a result of acidic $\mathrm{pH}[20]$. It has been observed, absence of peptidase and subtilase, subtilisin kexin isozyme-1 (SKI-1)/site 1 protease (S1P) that facilitates biochemical activity of glycoprotein cleavage attracts the rational for LASV vaccine and therapeutic production due to its infectivity as the essential surface functionality of the virion [21] The $L$ and $Z$ proteins are encoded by $L$ segment of RNA, unlike $S$ segment that encode nucleoprotein and glycoprotein [22]. L protein are majorly made up of RNA polymerase that is dependent at $\mathrm{C}$ domain and related to the viral nucleocapsid [23], and $\mathrm{N}$ domain function as transcription terminus for the virion through enzymatic processes of endonuclease [24].

The causative agent of virulent acute hemorrhagic fever, Lassa fever, was first discovered in Borno state, Nigeria, 1960 [5]. It has an incubation period of 6-21 days and characterized by symptoms such as fever, general weakness, and malaise. It has often associated with, headache, sore throat, muscle pain, chest pain, nausea, vomiting, diarrhea, and cough, few days after infection, follow by abdominal pain. Severe cases usually result to puffy face, pleural effusion, bleeding from different body orifice, gastrointestinal tract, and hypotension follow by death after 14 days of fatal cases [25]. The wide spread of LASV in Nigeria has been a subject of interest due to the increase in this deadly disease. According to the report by Agbonlahor et al, it was narrated that year 2012 recorded widest spread and higher incidence of Lassa 
hemorrhagic fever among different states in Nigeria. Edo, Delta, Ondo, Rivers, Ebonyi, Kano, Yobe, Benue, Kaduna, Kogi, Bauchi, Adamawa, Abia, Anambra, Imo States and the Federal Capital Territory, Abuja were affected states [26]. Following the surge of LASV endemicity in 2012, another marked incidence were recorded in Nigeria, 2018, with 394 confirmed cases in 19 states, and estimated 25\% cases led to death [9]. Increased in Lassa fever wide spread in Nigeria has posed a serious threat to the public health, with the recent outbreaks from January to February 2019. A total of 324 confirmed cases with 72 death cases were reported by Nigeria Centre for Disease Control (NCDC), while two separate case were reported in fifth week

with confirmed cases of 68 and week 6, 37 cases and 10 death cases were also reported [27]. This analysis aimed at acquiring the genomic variation of Lassa virus ancestral evolution with evolvement of new strains in different lineage and its geographic distributions through Bayesian inference, using genomic sequence across affected states in Nigeria and endemic countries in West Africa.

\section{Results}

\section{Homologous classification and genomic sequence flow of LASV in West Africa.}

Evolutionary relationship of Lassa mamarenavirus and other arenaviruses has revealed the distribution of different viruses across the globe. Basically, the phylogenetic analysis helped to classified the viruses into 3 distinct monophyletic groups, i.e. the old world arenaviruses, which were Lassa mammarenavirus, Mopeia virus AN20410, Morogoro mammarenavirus, Luna mammarenavirus, Mobala mammarenavirus, Ippy mammarenavirus, Merino Walk mammarenavirus, Lymphocytic choriomeningitis mammarenavirus and Lujo mammarenavirus, mainly from African countries, while the new world are Allpahuayo mammarenavirus, Flexal mammarenavirus. Cali mammarenavirus, Pirital mammarenavirus, Brazilian mammarenavirus, Paraguayan mammarenavirus, Cupixi mammarenavirus, Whitewater Arroyo mammarenavirus, Machupo mammarenavirus, Bear Canyon mammarenavirus, Argentinian mammarenavirus, Chapare mammarenavirus, Tacaribe mammarenavirus, Oliveros mammarenavirus, Guanarito mammarenavirus, Latino mammarenavirus and Tamiami mammarenavirus mostly found in North and south America28]. The other group were Reptarenaviruses, viruses has a host origin of reptiles e.g. Snake, and they are, University of Giessen virus, Golden Gate virus, ROUT virus, University of Helsinki virus, and CAS virus as shown in Figure 1.

GenBank and virus pathogen resource database (ViRP) recorded a total of 1,903 genomic sequences for LASV and 1,796 sample sequences from Africa. 347 are multimammate mouse (Mastomys natalensis) origin, 1,374 are from human host and 75 sequence are unknown source. The distribution of the sequences across endemic West African countries are shown in Figure 2.

Figure 1,Maximum likelihood tree of the Lassa Mammarenavirus nuclearprotein and 40 homologous sequences generated from ortho database and virussite databases. The evolutionary history was inferred 
by character-based method with Jones Taylor Thornton (JTT) matrix-based substitutional model. Pairwise distances were determine by routinely applying Neighbor-Join and BioNJ algorithms to the trees.

Figure 2, LASV genomic sequence distribution among West African countries (Guinea, Liberia, Nigeria, Sierra Leone, and Togo) as recorded in GenBank, from 1969 to 2018.

\section{Transcription Starting Site (Promoter) and TATA Box in LASV Genome}

Table 1, shows promoter sites of two Lassa mammarenavirus segments, $S$ and $L$ variants of the genome. The homologous gene MK291249.1 and MH157036.1 have the highest alignment scores with different strains isolated at different locations in Nigeria. The S segments started from position 2917 to 2947and L segments from 1863 to 1894 as predicted by promHG promoter prediction tool.

Table 1: Promoter site and TATA Box in LASV Genome.

\begin{tabular}{|l|c|l|l|l|}
\hline S/N & Segment & $\begin{array}{c}\text { Homologous } \\
\text { Gene }\end{array}$ & Predicted Promoter Site + TATA Box & $\begin{array}{c}\text { Position } \\
\text { (bp })\end{array}$ \\
\hline 1. & S_Segment & MK291249.1 & ATATAAACACCTGAGCTTAGTGGCCTTTCTG & $\begin{array}{l}2917 \\
2947\end{array}$ \\
\hline 2. & L_Segment & MH157036.1 & ATATAAACGTCTCAAAGAATGAATGATGTGGC & $\begin{array}{l}1863 \\
1894\end{array}$ \\
\hline
\end{tabular}

\section{Effective LASV siRNA}

Table 2, shows list of 10 Lassa mammarenavirus siRNA designed by BLOCK-iT RNAi Designer based on statistical analysis of valid siRNA and branded algorithm. The regions of each designed Oligos were represented as open reading frame (ORF) and $5^{\prime}$ untranslated region (5' UTR), the leader RNA, followed by the GC content of each siRNA were designed. BLAST results of highly species-specific gene for the designed RNAi DNA sequences shown in the last left column.

Table 2: LASV siRNA and targeted genes in humans. 


\begin{tabular}{|c|c|c|c|c|c|c|c|}
\hline \multirow[t]{2}{*}{$\mathbf{S} / \mathbf{N}$} & \multicolumn{3}{|c|}{ S_Segment (MK291249.1) } & \multicolumn{3}{|c|}{ L_Segment (MH157036.1) } & \multirow{2}{*}{$\begin{array}{c}\text { Targeted Genes by } \\
\text { BLAST Alignment }\end{array}$} \\
\hline & Sequence(DNA) & Region & GC\% & Sequence(DNA) & Region & GC\% & \\
\hline 1. & GCTACAAACTCTAGAGCTA & 5’UTR & 42.11 & CCATTGAACTCTTTGTCTT & ORF & 36.85 & $\begin{array}{l}\text { NM_004446 } \\
\text { CD742789 }\end{array}$ \\
\hline 2. & GCTAACCACTGTGGGACTA & 5’UTR & 52.64 & CCACAAACCCAGATGCTAT & ORF & 47.37 & $\begin{array}{l}\text { CR933660 } \\
\text { NM_004446 }\end{array}$ \\
\hline 3. & GCAAGCAGACAACATGATA & 5’UTR & 42.11 & GCTAAGTGCTTCAGAATTA & ORF & 36.85 & $\begin{array}{l}\text { NM_030625 } \\
\text { BX649078 }\end{array}$ \\
\hline 4. & GCATATGGCATAGATCTTT & ORF & 36.85 & GCACAACATTCCTTACTTA & ORF & 36.85 & $\begin{array}{l}\text { CA392182 } \\
\text { NM_030625 }\end{array}$ \\
\hline 5. & CCATGAGAATATTTGGCAT & ORF & 36.85 & GCATAACACTTTGAGCATT & ORF & 36.85 & $\begin{array}{l}\text { AK129490 } \\
\text { NM_001402 }\end{array}$ \\
\hline 6. & GCATACAAGCTCCAGCTTT & ORF & 47.37 & GCACCTTACAACCTGGTAT & ORF & 47.37 & $\begin{array}{l}\text { NR_002728 Al873453 } \\
\text { NM_138459 }\end{array}$ \\
\hline 7. & CCTAACAACTCCGTCTCTT & ORF & 47.37 & GCAAGGAACCTATCACCAT & ORF & 47.37 & \\
\hline 8. & GCTGCTGTGTACTCAAATT & ORF & 42.11 & GCTTGTCAGTTAGAACATT & ORF & 36.85 & XR_109175 \\
\hline 9. & GCAGGTCATCTGAGGTCAA & ORF & 52.64 & CCAACAGACTCCAAATCAT & ORF & 42.11 & NM_030625 \\
\hline 10. & GCATTAAACGCTGCACATT & ORF & 42.11 & GCTAACTTCTGTCTTGATA & ORF & 36.85 & $\begin{array}{l}\text { AK125883 } \\
\text { NM_004446 }\end{array}$ \\
\hline
\end{tabular}

\section{Phylogenies and discrete phylogeography}

Based on comprehensive genomic sequence evaluation, it was recorded from GenBank, a total estimation of LASV sequences from affected 16 states in Nigeria between years 2008 to 2018 to be 735 . Estimated sequences were grouped into two regions of Nigeria (North and South); the Northern states were Bauchi = 33 , Benue $=3$, Kaduna $=3$, Kogi $=22$, Nassarawa $=28$, Plateau $=17$, and Taraba $=11$, while southern states include Anambra $=9$, Delta $=18$, Ebonyi $=107$, Edo $=241$, Ekiti $=4$, Enugu $=43$, Imo $=10$, Ondo $=$ 182, and Rivers $=4$, as shown in Figure 3 .

Figure 3: LASV genomic sequence distribution among different affected states in Nigeria from 2008 to 2018 as it was recorded by GenBank. Figure 4: circular phylogenetic tree of LASV S-segment genome showing recently isolated strains in different lineage.

Figure 4, shows the circular phylogenetic tree of LASV S-segment genome inferred by Maximum-Likelihood phylogeny with General Time Reversible (GTR) substitution model and Gamma Distributed Invariant (G+I) 
rates among sites in MEGA-X software and visualized with iTOL online tool. LASV lineage I to VI were indicated with different color ranges and newly 75 sequenced segments were represented in red color.

From the MCC phylogenetic tree in Figure 5, it can be inferred the domestic relationship of LASV in different states represented by the tree branches and nodes. The strains from Bauchi, Benue, Plateau, Nasarawa and Kaduna from same clades shows distinct evolutionary relationship, comparing the nodes. Most of the sampled strains in southern part of the country were shown to evolve from two major monophyletic group; Ebonyi, Edo, Delta and Anambra which form the first clade and second clade, Ondo Kogi and Ekiti from the second one. The strains from Plateau and Rivers were seen to be genetically distributed across different clades.

Figure 5: Maximum clade credibility (MCC) phylogenies of selected LASV isolates, from states in Nigeria. MCC phylogenies of LASV (LASVsSgp1) among the affected states in Nigeria. Descendant nodes and branches are colored according to the most probable location. Year before recent analysis are shown by the scale bar in the bottom of the tree.

With the above MCC tree, the spread of LASV in Nigeria can be understand using the annotated tree to determine the spatiotemporal distribution on Google Earth using KML file generated with SPREAD as shown in Figure 6.

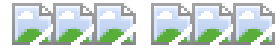

Figure 6: Phylogeographic reconstruction of LASV showing spatiotemporal dispersion among different states in Nigeria, through Google Earth with KML file Generated in SPREAD.

\section{Discussion}

The arenaviruses are majorly transmitted by rodent host and some species by reptiles, causing different human pathogenic diseases. The single stranded ambisens RNA virus were classified into old world arenavirus, new world arenavirus and reptarenavirus based on phylogenetic classification in Figure 1, they are predominantly found in Africa, South America and other parts of the world. The old and new world arenaviruses are taxonomically grouped into one genera, the Mamarenavirus, while the reptile-host viruses from the outer group of homologous classification are Reptarenavirus. The human pathogenic infections of old world arenavirus include severe Lassa fever and mild Lymphocytic choriomeningitis caused by Lassa mammarenavirus and Lymphocytic choriomeningitis mammarenavirus respectively, no human infection has been recoded for other viruses in the group. Importantly, several reasserting studies using Mopeia virus for the development of attenuated LASV vaccines was reported by Johnson et al, [29] and Moshkoff et al, [30]. Among the new world arenaviruses, the genomic sequence distribution across different West African endemic countries were shown in Figure 2. It was recorded, Nigeria has the highest sampled sequence in GenBank, follow by Sierra Leone, Liberia, Guinea, and Togo. This was only recorded for countries where there was substantial outbreak, while countries where there were few cases, periodic isolation, and serological evidences are not included. Among the new world arenaviruses, Brazilian 
mammarenavirus, Machupo mammarenavirus, Argentinian mammarenavirus, and Guanarito mammarenavirus found in Brazil, Bolivia, Argentina and Venezuela respectively were transmitted by rodent host and known to cause hemorrhagic fever in human $[35,38]$. The predicted small interfering RNA revealed the potential of the down regulation activities of some human genes through specific base paring. Müller and Günther reported siRNA targeting upper stream of $S$ and $L$ segment are capable of down regulating reporter gene expressing LASV mRNA construct and replicon [31].

Importantly, LASV genetic makeup has been characterized on the genotypic differences of ancestral evolution within varying time of outbreaks. Similar to the study is the report of Kafetzopoulou et al [32], which highlighted the lineage into six groups ( $($ to $\mathrm{VI})$ as shown in Figure 4. Most of the sequence samples were fall within lineage II, one strain in lineage III and strain suspected to be newly emerging due to the divergence from lineage I. Based on phylogenetic analysis, LASV sequence obtained from different part of Nigeria were genetically related and grouped into distinct clades according to geographical location from the reconstructed time scale MCC tree in Figure 5. Due to the incomplete sequence reported, one of the location in north central part of the country (Abuja) was not covered by the analysis, this restriction was measured not to affect other states. Tracing the spread of LASV by the vector, Mastomys natalensis from previous to recent outbreaks, we observe the phylogeography in relation to the geopolitical zones and time of isolates. It was suggested, Benue, Imo, and Bauchi states represent the host etiology of the LASV, because of the root origin of the branches, time of common ancestors and dominancy of monophyletic group, while the spread across other neighboring states were based on genetic pedigree dated to previous outbreaks as at year 2008 to 2012. Benue state is one out of the six state in eastern Middle Belt of Nigeria. It share boundaries with Cross River state on the south through Yala Local Government Area, Enugu and Ebonyi states. Benue also border with Kogi and Nassawara state on the east and north respectively, and on the northeast by Taraba state. Ingestion of cooked rat meat is a common practice among the people of Benue state, but not raw or undercooked meat according to Olusi et al, [33]. Contrary to the previous outbreaks, phylogeography of the recent strains identified spontaneous spreads of LASV from south-west to south-south and north central through the states that shared common border. This finding shared similar opinion with recent the recent study by Ehichioya et al, [34], confirmed that, majority of the sampled strained in lineage II evolved from Ondo state, a south-western state bordering with Kogi (north-central) and Edo (south-south).

\section{Conclusion}

The geography of recent transmission from year 2017 to 2019 indicates vectors were fast spreading LASV from Ondo states to Delta, Edo, and Kogi states, while spread across north eastern states suggests a vector origin from Bauchi state. From the result of this study, we suggests, federal government should initiate a vector surveillance program to curtails further spread of LASV, especially, states bordering with north western states and north central. The study evidently outlined the path of transmission based on genetic homology of the sampled LASV sequences in affected geographical locations through spatial phylogenetic reconstruction of evolutionary dynamics. 


\section{Methods}

\section{LASV homologs and genomic sequence distribution}

To determine the homologous recombination of the LASV and other arenaviruses through DNA sequence phylogeny, we collected homologous sequences of arenaviruses from orthology database, OrthoDB V10 [35] and viral genome database, viruSITE [36]. Following sequence collection, sequences were manually edited, aligned using multiple sequence alignment program, ClustalW and used to construct phylogenetic tree in MEGA X software [37]. An online tool (Interactive tree of life: iTol) was used to annotate and display the tree [38]. According to Centers for Disease Control and Prevention (CDC), LASV was endemic in West Africa countries of Nigeria, Guinea, Sierra Leone, Liberia, Mali, Côte d'Ivoire, and new strains were recently found in Togo, 2016 [3]. To determine the genomic diversity of LASV strains, a distribution test was used to measure the sequence flow among the endemic countries and states in Nigeria. This was estimated using virus pathogen resource database, ViRP [39] and GenBank [40]. The data sequences of LASV nucleoprotein (LASVsSgp1) gene among the affected states from Nigeria within a period of 2008 to 2019 and endemic countries of West Africa with isolation date ranging from 1969 to 2018 were analyzed. For LASV promoter sites and effective short interference RNA (siRNA) in LASV genome, PromH online promoter site prediction tool was used to predict accurate Transcription Starting Sites (TSS) with TATA box [41], while siRNA were designed by BLOCK-iT ${ }^{\text {TM }}$ RNAi Designer [42].

\section{Genomic Variation and Phylogeographic Distribution in Nigeria}

Genomic variation and phylogeographic distribution, of LASV was analyzed using the S segment of the genomic sequences generated by Irrua Specialist Teaching Hospital (ISTH) in collaboration with Institute for Lassa Fever Research and Control (ILFRC), Irrua, Edo State, published in GenBank. A total of 499 LASV sequence samples were analysed. 354 out of the sequences were real-time LASV sequences in Nigeria and other west Africa countries recorded by Kafetzopoulou et al, [32], while 70 were collected from GenBank with keyword (txid11620[Organism]) to select appropriate accession number of S segment with the collection date ranging from 2008 to 2018, while the additional 75 sequences were recently laboratoryconfirmed Lassa fever patients from Edo, Ondo, Kogi, Delta, Ebonyi and Benue States with dates of onset from the end of December 2018 to March 2019 reported to virological database [43]. Maximum-likelihood phylogenetic analysis was performed on coding sequence of $S$ segment to determine the lineage of recent LASV samples.

Prior to BEAST setup [44], sequence data were aligned with ClustalW in MEGA-X [37] and exported in Nexus format. Using BEAUti program, the nexus file was imported, subsequently, date, site model, clock model, priors, discrete trait and Markov chain Monte Carlo (MCMC) were set up accordingly for Maximum Clade Credibility (MCC) tree and discrete phylogeography. BEAST 2.5 tool was used to run $\mathrm{xml}$ file generated from BEAUti program for 6 hours for maximum Effective Sample Size (ESS). The Trace log and dot trees files generated were further sampled using Tracer v1.7.1 [45] and TreeAnnotator program for MCMC estimation and annotation of MCC trees at $95 \%$ highest probability density (HPD). Annotated MCC trees 
were constructed and visualized with FigTree v1.4.4. In summary, spatiotemporal transmission routes of LASV in Nigeria was visualized with Google Earth Pro v7.3.2 tool after configuration of geographical locations (latitude and longitude) in SPREAD v1.0.6 [46] software, followed by conversion of MCC trees into a keyhole markup language $(\mathrm{KML})$ file. To certify the reproducibility of end result, this methodology was guided by the preceding study on norovirus in China [47], and influenza A H5N1 virus in Egypt [48].

\section{List Of Abbreviations}

BEAST: Bayesian Evolutionary Analysis Sampling TreesBP: Base Pare ER: Endoplasmic ReticulumESS: Effective Sample Size GP: glycoprotein GTR: General Time Reversible HPD: Highest Probability Density IFN: Interferon I-FP: Internal Fusion Loop/LFRC: Institute for Lassa Fever Research and Control ISTH: Irrua Specialist Teaching Hospital KML: Keyhole Markup LanguageLASV: Lassa virusMCC: Maximum Clade Credibility MCMC: Markov chain Monte Carlo NCDC: Nigeria Centre for Disease ControlORF: Reading FrameS1P: Site 1 ProteasesiRNA: Small interfering RNASKI-1: Subtilisin Kexin Isozyme-1TSS:

Transcription Starting SiteViRP: Virus Pathogen Resource Database

\section{References}

1.Yun NE, Walker DH: Pathogenesis of Lassa fever. Viruses 2012, 4(10):2031-2048.

2.Safronetz D, Lopez JE, Sogoba N, Traore SF, Raffel SJ, Fischer ER, Ebihara H, Branco L, Garry RF, Schwan TG et al: Detection of Lassa virus, Mali. Emerging infectious diseases 2010, 16(7):1123-1126.

3.Whitmer SLM, Strecker T, Cadar D, Dienes HP, Faber K, Patel K, Brown SM, Davis WG, Klena JD, Rollin PE et al: New Lineage of Lassa Virus, Togo, 2016. Emerging infectious diseases 2018, 24(3):599-602.

4.Gunther S, Lenz O: Lassa virus. Critical reviews in clinical laboratory sciences 2004, 41(4):339-390.

5.Andersen KG, Shapiro BJ, Matranga CB, Sealfon R, Lin AE, Moses LM, Folarin OA, Goba A, Odia I, Ehiane PE et al: Clinical Sequencing Uncovers Origins and Evolution of Lassa Virus. Cell 2015, 162(4):738-750.

6.Qi X, Wang W, Dong H, Liang Y, Dong C, Ly H: Expression and X-Ray Structural Determination of the Nucleoprotein of Lassa Fever Virus. Methods in molecular biology (Clifton, NJ) 2018, 1604:179-188.

7.Loureiro ME, D'Antuono A, Lopez N: Virus(-)Host Interactions Involved in Lassa Virus Entry and Genome Replication. Pathogens (Basel, Switzerland) 2019, 8(1).

8.Ly H: Differential Immune Responses to New World and Old World Mammalian Arenaviruses. International journal of molecular sciences 2017, 18(5).

9.Siddle KJ, Eromon P, Barnes KG, Mehta S, Oguzie JU, Odia I, Schaffner SF, Winnicki SM, Shah RR, Qu J et al: Genomic Analysis of Lassa Virus during an Increase in Cases in Nigeria in 2018. The New England journal of medicine 2018, 379(18):1745-1753. 
10.Zhang Y, Li L, Liu X, Dong S, Wang W, Huo T, Guo Y, Rao Z, Yang C: Crystal structure of Junin virus nucleoprotein. The Journal of general virology 2013, 94(Pt 10):2175-2183.

11.Pattis JG, May ER: Influence of RNA Binding on the Structure and Dynamics of the Lassa Virus Nucleoprotein. Biophysical journal 2016, 110(6):1246-1254.

12. Hastie KM, Liu T, Li S, King LB, Ngo N, Zandonatti MA, Woods VL, Jr., de la Torre JC, Saphire EO: Crystal structure of the Lassa virus nucleoprotein-RNA complex reveals a gating mechanism for RNA binding. Proceedings of the National Academy of Sciences of the United States of America 2011, 108(48):1936519370.

13.Brunotte L, Kerber R, Shang W, Hauer F, Hass M, Gabriel M, Lelke M, Busch C, Stark H, Svergun DI: Structure of the Lassa virus nucleoprotein revealed by X-ray crystallography, small-angle X-ray scattering, and electron microscopy. Journal of Biological Chemistry 2011:jbc. M111. 278838.

14.Brunotte L, Kerber R, Shang W, Hauer F, Hass M, Gabriel M, Lelke M, Busch C, Stark H, Svergun DI et al: Structure of the Lassa virus nucleoprotein revealed by $X$-ray crystallography, small-angle $X$-ray scattering, and electron microscopy. The Journal of biological chemistry 2011, 286(44):38748-38756.

15.Carnec X, Baize S, Reynard S, Diancourt L, Caro V, Tordo N, Bouloy M: Lassa virus nucleoprotein mutants generated by reverse genetics induce a robust type I interferon response in human dendritic cells and macrophages. Journal of virology 2011, 85(22):12093-12097.

16.Zhang Y, Chen H, Han JG: Insight into the binding modes of Lassa nucleoprotein complexed with ssRNA by molecular dynamic simulations and free energy calculations. Journal of biomolecular structure \& dynamics 2015, 33(5):946-960.

17.Hastie KM, Saphire EO: Lassa virus glycoprotein: stopping a moving target. Current opinion in virology 2018, 31:52-58.

18.Schlie K, Maisa A, Lennartz F, Stroher U, Garten W, Strecker T: Characterization of Lassa virus glycoprotein oligomerization and influence of cholesterol on virus replication. Journal of virology 2010, 84(2):983-992.

19.Galan-Navarro C, Rincon-Restrepo M, Zimmer G, Ollmann Saphire E, Hubbell JA, Hirosue S, Swartz MA, Kunz S: Oxidation-sensitive polymersomes as vaccine nanocarriers enhance humoral responses against Lassa virus envelope glycoprotein. Virology 2017, 512:161-171.

20.Willard KA, Alston JT, Acciani M, Brindley MA: Identification of Residues in Lassa Virus Glycoprotein Subunit 2 That Are Critical for Protein Function. Pathogens (Basel, Switzerland) 2018, 8(1).

21.Amanat F, Duehr J, Oestereich L, Hastie KM, Ollmann Saphire E, Krammer F: Antibodies to the Glycoprotein GP2 Subunit Cross-React between Old and New World Arenaviruses. mSphere 2018, 3(3). 
22.Brunotte L, Lelke M, Hass M, Kleinsteuber K, Becker-Ziaja B, Gunther S: Domain structure of Lassa virus L protein. Journal of virology 2011, 85(1):324-333.

23.Vieth S, Torda AE, Asper M, Schmitz H, Gunther S: Sequence analysis of L RNA of Lassa virus. Virology 2004, 318(1):153-168.

24.Lehmann M, Pahlmann M, Jerome H, Busch C, Lelke M, Gunther S: Role of the C terminus of Lassa virus L protein in viral mRNA synthesis. Journal of virology 2014, 88(15):8713-8717.

25.Lassa fever/https://www.who.int/news-room/fact-sheets/detail/lassa-fever]

26.Agbonlahor DE, Erah A, Agba IM, Oviasogie FE, Ehiaghe AF, Wankasi M, Eremwanarue OA, Ehiaghe IJ, Ogbu EC, Iyen RI et al: Prevalence of Lassa virus among rodents trapped in three South-South States of Nigeria. Journal of vector borne diseases 2017, 54(2):146-150.

27.Lassa Fever-Nigeria: Emergencies preparedness, response /https://www.who.int/csr/don/14-february2019-lassa-fever-nigeria/en/]

28.Old World/New World Arenaviruses [https://www.cdc.gov/vhf/virus-families/arenaviruses.html] 29.Johnson DM, Jokinen JD, Lukashevich IS: Attenuated Replication of Lassa Virus Vaccine Candidate ML29 in STAT-1(-/-) Mice. Pathogens (Basel, Switzerland) 2019, 8(1).

30.Moshkoff DA, Salvato MS, Lukashevich IS: Molecular characterization of a reassortant virus derived from Lassa and Mopeia viruses. Virus genes 2007, 34(2):169-176.

31.Muller S, Gunther S: Broad-spectrum antiviral activity of small interfering RNA targeting the conserved RNA termini of Lassa virus. Antimicrobial agents and chemotherapy 2007, 51(6):2215-2218.

32.Kafetzopoulou LE, Pullan ST, Lemey P, Suchard MA, Ehichioya DU, Pahlmann M, Thielebein A, Hinzmann J, Oestereich L, Wozniak DM et al: Metagenomic sequencing at the epicenter of the Nigeria 2018 Lassa fever outbreak. Science (New York, NY) 2019, 363(6422):74-77.

33.T. A. Olusi et al.: Antibodies to Toxoplasma gondii in a rat-eatingpopulation of Benue State, Nigeria. Annals of Tropical Medicine \& Parasitology 1994, 88(2):217-218.

34.Ehichioya DU, Dellicour S, Pahlmann M, Rieger T, Oestereich L, Becker-Ziaja B, Cadar D, Ighodalo Y, Olokor T, Omomoh E et al: Phylogeography of Lassa virus in Nigeria. Journal of virology 2019.

35.Kriventseva EV, Kuznetsov D, Tegenfeldt F, Manni M, Dias R, Simao FA, Zdobnov EM: OrthoDB v10: sampling the diversity of animal, plant, fungal, protist, bacterial and viral genomes for evolutionary and functional annotations of orthologs. Nucleic acids research 2019, 47(D1):D807-d811.

36.Stano M, Beke G, Klucar L: viruSITE-integrated database for viral genomics. Database: the journal of biological databases and curation 2016, 2016. 
37.Kumar S, Stecher G, Li M, Knyaz C, Tamura K: MEGA X: Molecular Evolutionary Genetics Analysis across Computing Platforms. Molecular biology and evolution 2018, 35(6):1547-1549.

38.Letunic I, Bork P: Interactive tree of life (iTOL) v3: an online tool for the display and annotation of phylogenetic and other trees. Nucleic acids research 2016, 44(W1):W242-245.

39.Pickett BE, Sadat EL, Zhang Y, Noronha JM, Squires RB, Hunt V, Liu M, Kumar S, Zaremba S, Gu Z et al: ViPR: an open bioinformatics database and analysis resource for virology research. Nucleic acids research 2012, 40(Database issue):D593-598.

40.Clark K, Karsch-Mizrachi I, Lipman DJ, Ostell J, Sayers EW: GenBank. Nucleic acids research 2016, 44(D1):D67-72.

41.Solovyev VV, Shahmuradov IA: PromH: Promoters identification using orthologous genomic sequences. Nucleic acids research 2003, 31(13):3540-3545.

42.BLOCK-iT ${ }^{T M}$ RNAi Designer /https://rnaidesigner.thermofisher.com/rnaiexpress/setOption.do? designOption $=$ sirna\&pid $=-2159752509464319169]$

43.2019 Lassa virus sequencing in Nigeria - Final field report (75 samples) [http://virological.org/]

44.Bouckaert R, Heled J, Kuhnert D, Vaughan T, Wu CH, Xie D, Suchard MA, Rambaut A, Drummond AJ: BEAST 2: a software platform for Bayesian evolutionary analysis. PLoS computational biology 2014, 10(4):e1003537.

45.Rambaut A, Drummond AJ, Xie D, Baele G, Suchard MA: Posterior Summarization in Bayesian Phylogenetics Using Tracer 1.7. Systematic Biology 2018, 67(5):901-904.

46.Bielejec F, Baele G, Vrancken B, Suchard MA, Rambaut A, Lemey P: SpreaD3: Interactive Visualization of Spatiotemporal History and Trait Evolutionary Processes. Molecular biology and evolution 2016, 33(8):2167-2169.

47.Qiao N, Ren H, Liu L: Genomic diversity and phylogeography of norovirus in China. BMC medical genomics 2017, 10(Suppl 3):51.

48.Scotch M, Mei C, Makonnen YJ, Pinto J, Ali A, Vegso S, Kane M, Sarkar IN, Rabinowitz P: Phylogeography of influenza A H5N1 clade 2.2.1.1 in Egypt. BMC genomics 2013, 14:871.

\section{Declarations}

\section{Acknowledgements}

Not applicable 


\section{Authors' contributions}

YAA designed the study, draft the manuscript and generate the data. YAA and YA analyzed the data. YA reviewed the manuscript. Both author read and approved the final manuscript.

\section{Funding}

Not applicable

\section{Availability of Data and Materials}

Datasets generated and analyzed during the study are included within the article.

\section{Competing interests}

Authors of this article affirm that they have no competing interests.

\section{Ethics approval and consent to participate}

Not applicable

\section{Consent for publication}

Not applicable

\section{Figures}




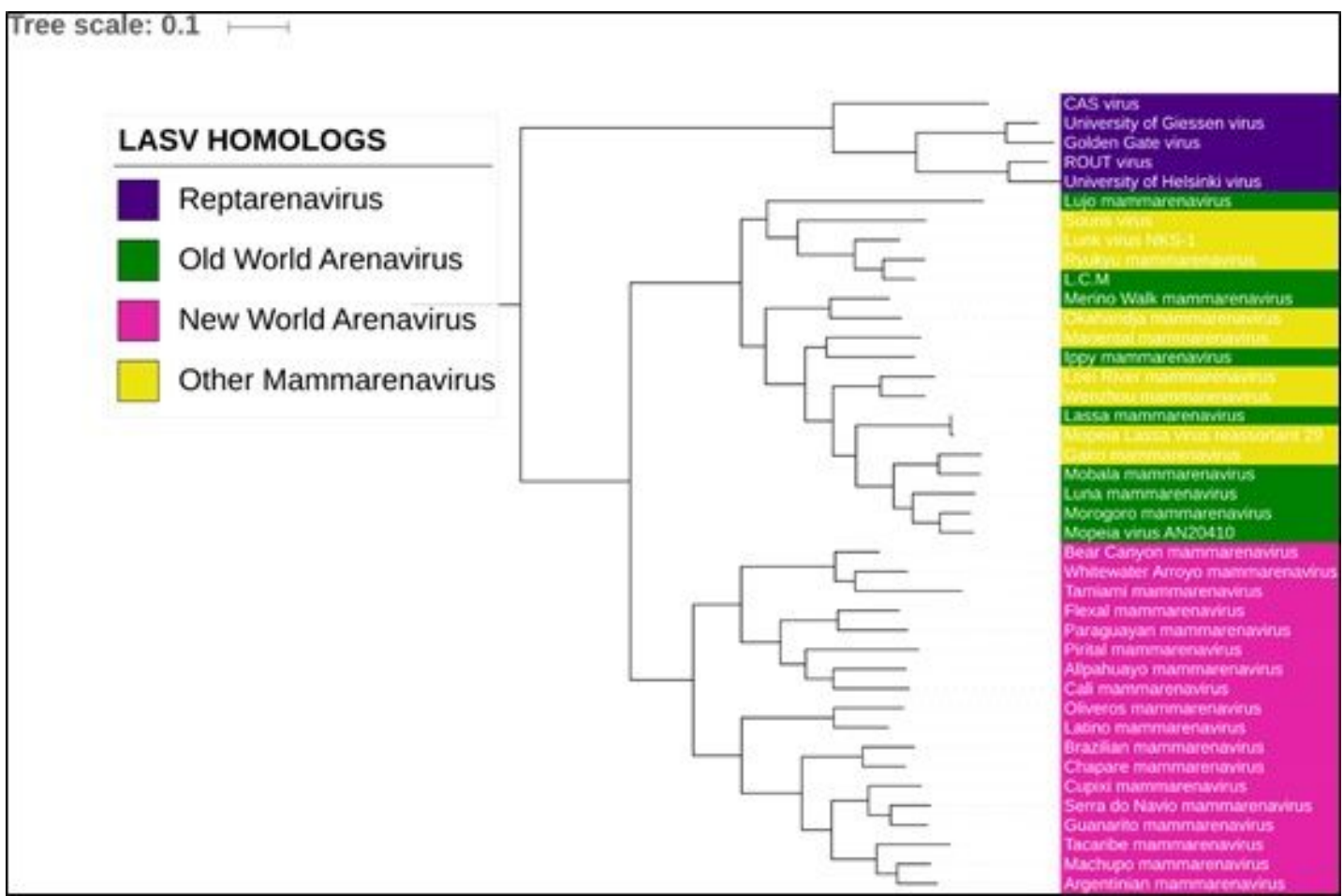

\section{Figure 1}

Maximum likelihood tree of the Lassa Mammarenavirus nuclearprotein and 40 homologous sequences generated from ortho database and virussite databases. The evolutionary history was inferred by character-based method with Jones Taylor Thornton (JTT) matrix-based substitutional model. Pairwise distances were determine by routinely applying Neighbor-Join and BioNJ algorithms to the trees.

\section{Lassa Virus Genomic Sequence Flow Chart Available in GenBank}

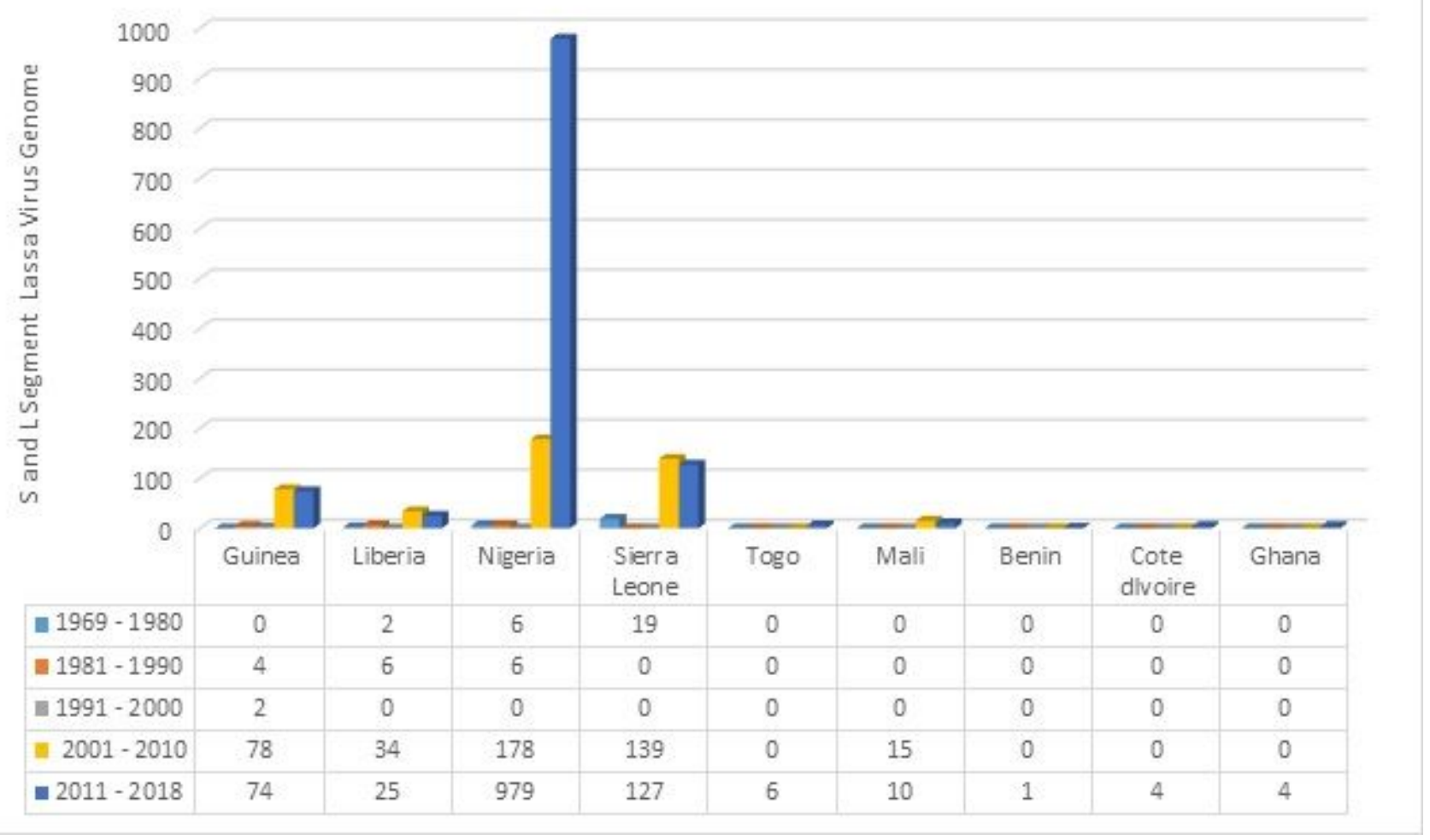


Figure 2

LASV genomic sequence distribution among West African countries (Guinea, Liberia, Nigeria, Sierra Leone, and Togo) as recorded in GenBank, from 1969 to 2018.

\section{Sampled sequence of Lassa virus distributions in Nigeria}

\begin{tabular}{|c|c|c|c|c|c|c|}
\hline - Anambra " Bauchi & a Benue & w Delta & = Ebonyi & $\equiv$ Edo & $=$ Ekiti & $=\mathrm{Imo}$ \\
\hline Ondo & Plateu & Divers & - Taraba & Enugu & - Osun & - Calabar \\
\hline
\end{tabular}

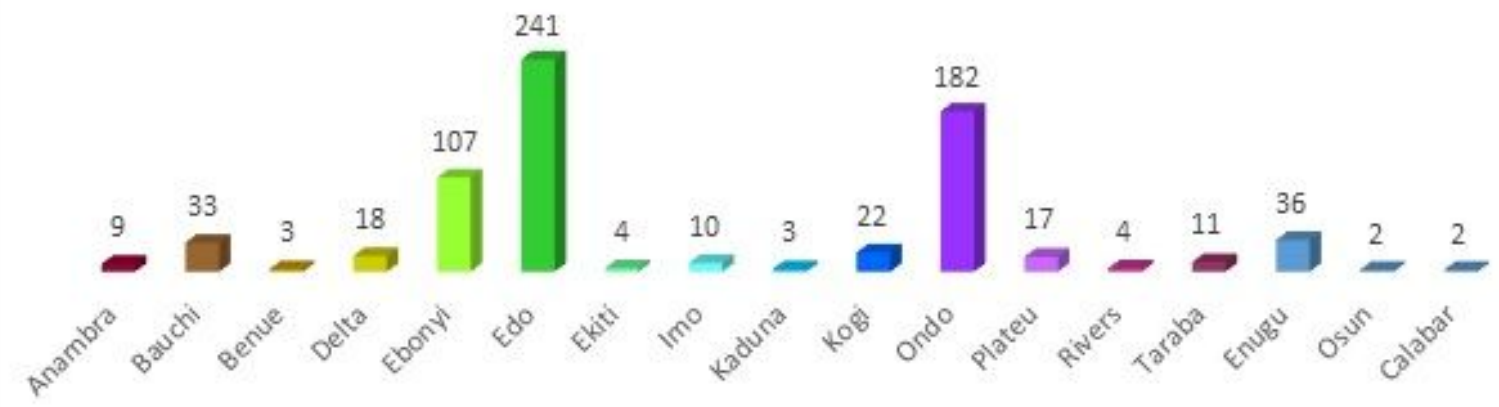

\section{Figure 3}

LASV genomic sequence distribution among different affected states in Nigeria from 2008 to 2018 as it was recorded by GenBank.

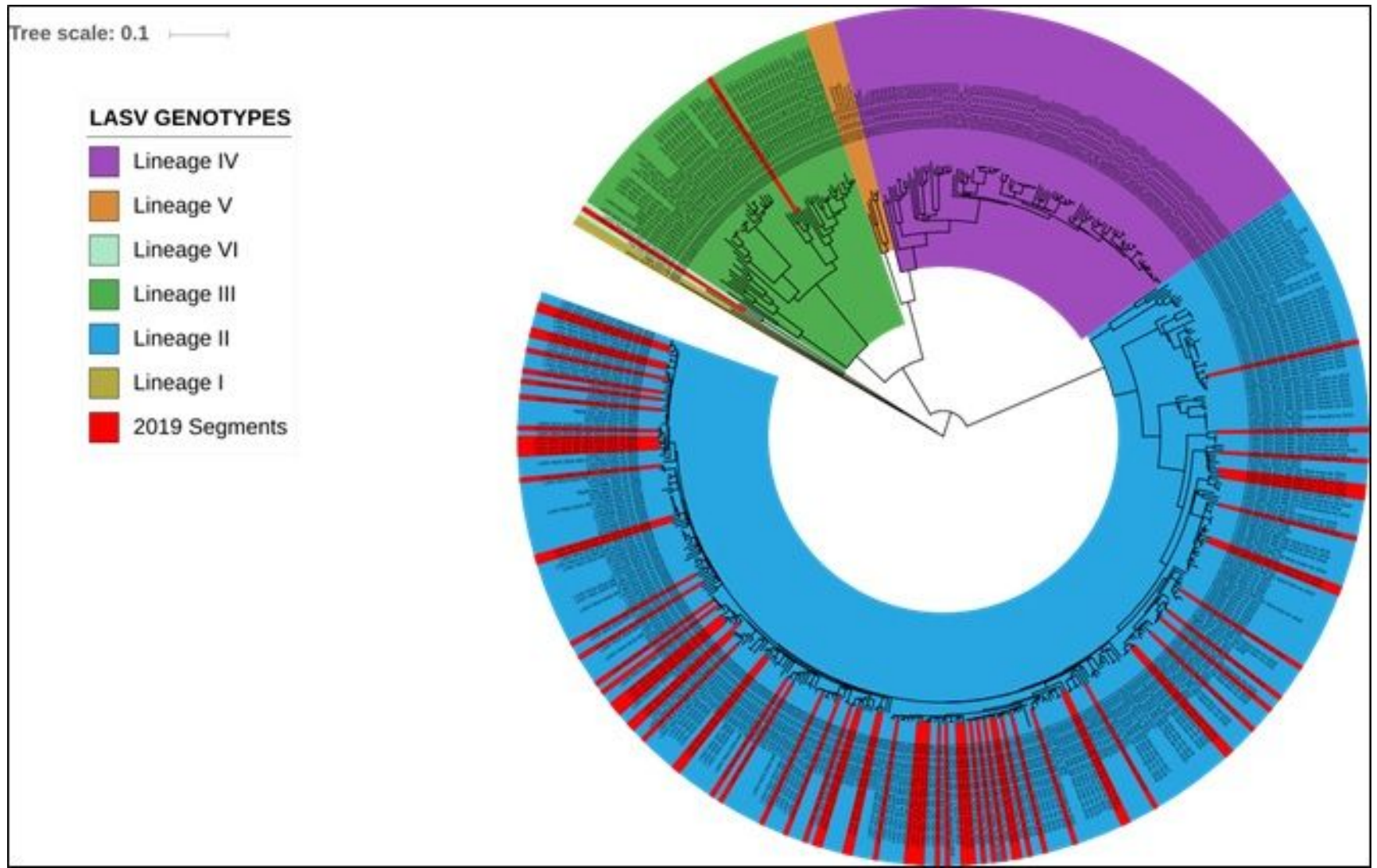


Figure 4

circular phylogenetic tree of LASV S-segment genome showing recently isolated strains in different lineage.

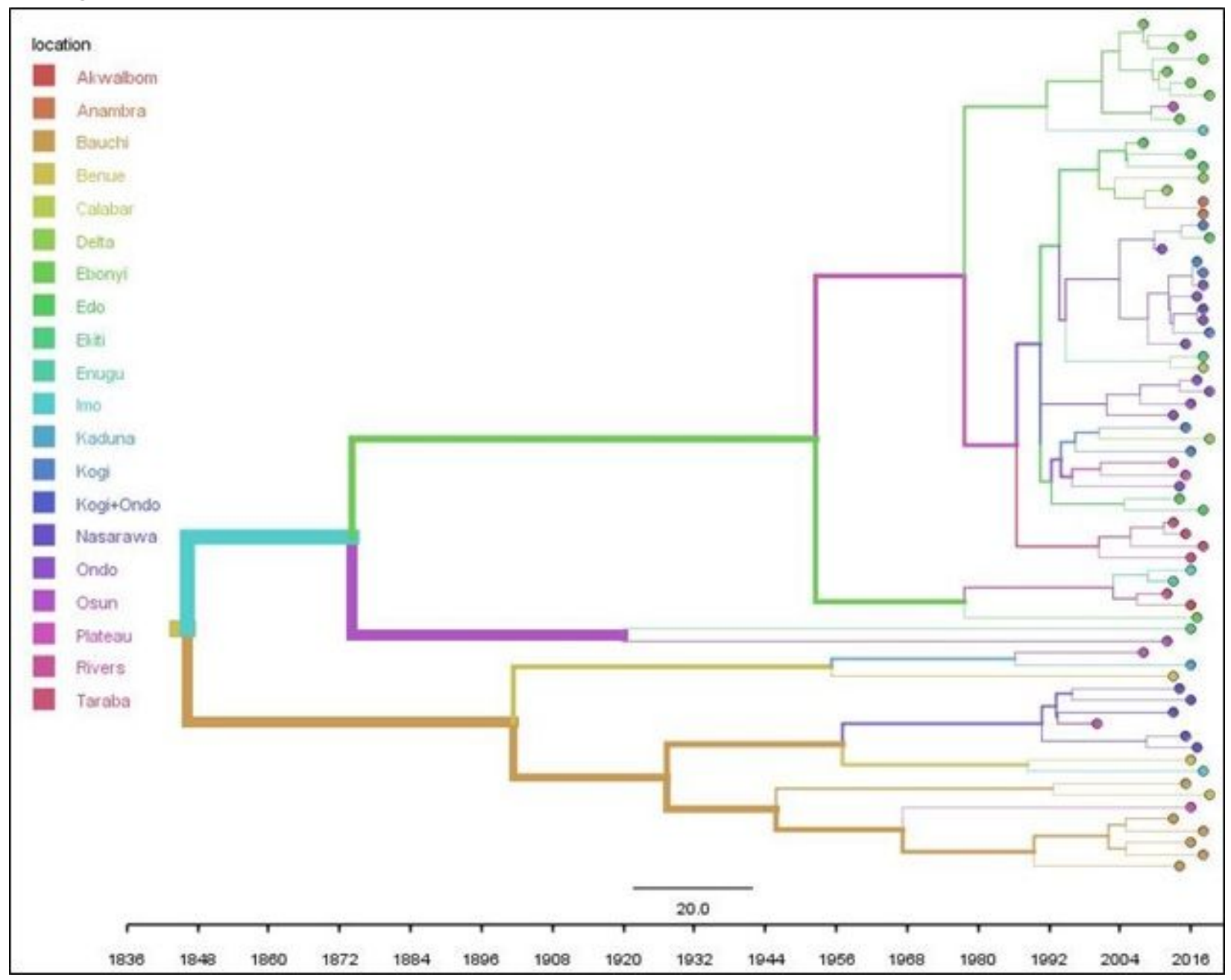

Figure 5

Maximum clade credibility (MCC) phylogenies of selected LASV isolates, from states in Nigeria. 

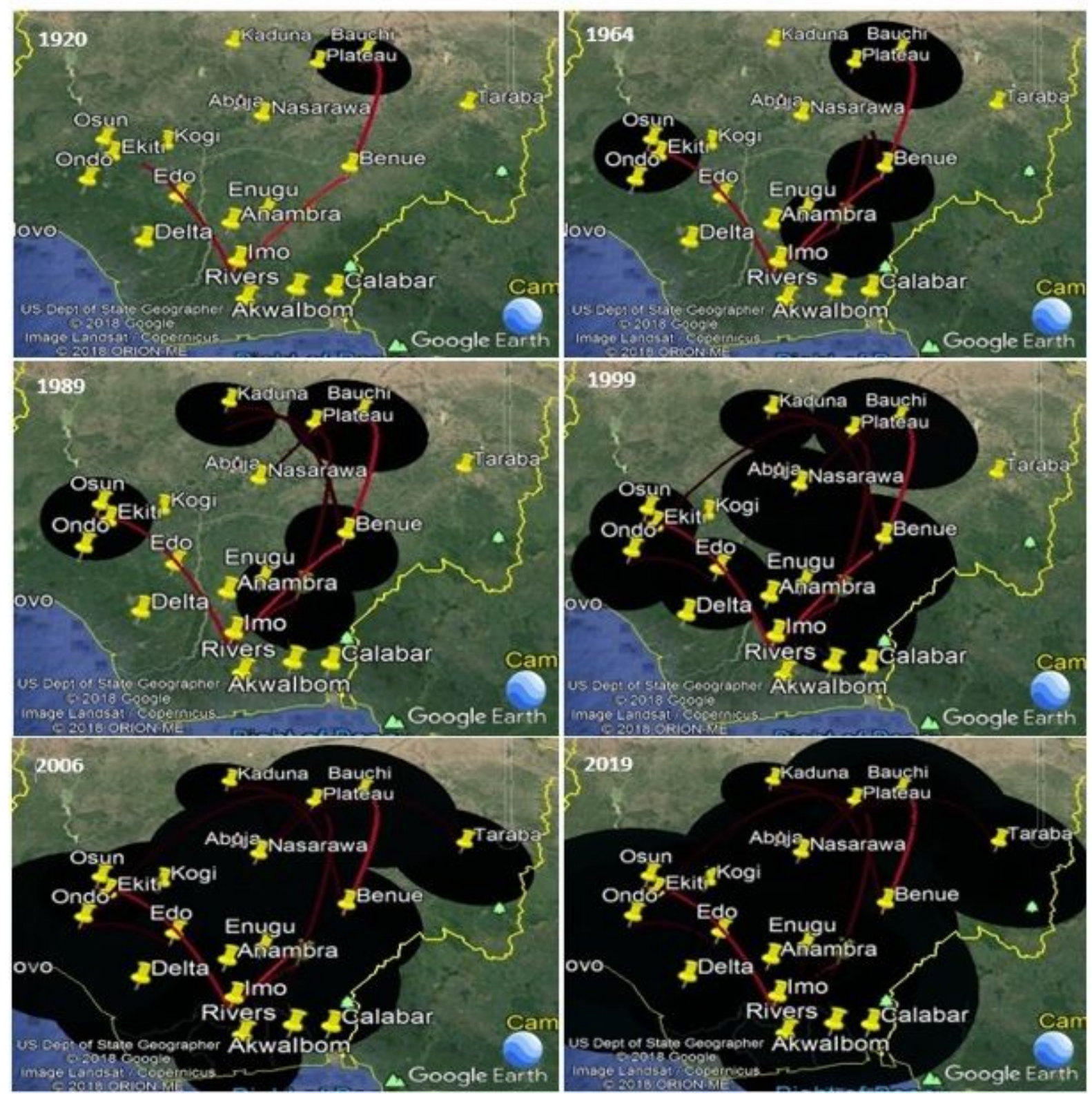

\section{Figure 6}

Phylogeographic reconstruction of LASV showing spatiotemporal dispersion among different states in Nigeria, through Google Earth with KML file Generated in SPREAD.

\section{Supplementary Files}

This is a list of supplementary files associated with this preprint. Click to download.

- Additionalfile6.pdf

- Additionalfile3.txt

- Additionalfile1.txt

- Additionalfile5.xlsx 
- Additionalfile2.xlsx

- Additionalfile4.txt 